\title{
Practical experiences on a real pumping system emulated by a hardware model and used as a remote laboratory
}

\author{
Jorge Pomares, Iván Perea, Carlos A. Jara, Francisco A. Candelas, Fernando Torres \\ University of Alicante, Carretera de San Vicente del Raspeig, s/n, PO Box 03690, Alicante \\ Spain (e-mail: \{jpomares, ivan.perea, carlos.jara, francisco.candelas, fernando.torres\}@ua.es)
}

\begin{abstract}
This paper describes a remote laboratory employed in the practical learning of the subject "Control Systems" which is taught in the Automatics and Robotics Master's degree at the University of Alicante. The application developed allows students to practice remotely different theoretical concepts using of a hardware model of a real industrial process about a pumping system. In the paper, it is described the most important features of the remote laboratory, highlighting its ability to perform the automatic evaluation of the student. The application proposes a set of practical experiences which students must resolve making use of the remote laboratory. In addition, the application offers a feedback that guides the student about the concepts to improve in their learning. This information can be used by the student to perform a self-learning. The paper ends with a study that describes the educational impact about the use of this tool in the students' learning.
\end{abstract}

Keywords: control education, distance learning, remote laboratory, self-learning

\section{INTRODUCTION}

With the arrival of the ESHE (European Space of Higher Education) (Clausen, 2005), university teachers must teach subjects taking into account two fundamental objectives: the acquisition of skills and the student's centred teaching. The student stops being a simple observer in the traditional model of master classes and becomes the main participant of their learning. One of the guidelines determined by ESHE is that traditional education must be combined with distance education, because a good distance teaching education provides flexibility to students. In this new educational context, and in the field of control education, virtual and remote laboratories (Jara et al., 2009; Jara et al, 2011) play an important role, because they are designed for an independent learner and/or a distance self-learning. The work presented in this paper shows the successful application of a remote laboratory used in the practical learning of the subject "Control Systems" taught in the Automatics and Robotics Master's degree at the University of Alicante. The application allows students to practice remotely different theoretical concepts using of a hardware model of a real industrial process about a pumping system.

The main objective to reach by the remote laboratory is that students can implement, monitor and verify control and automation techniques from home as if they were in the industrial plant. This overall objective is specified in the following items:

- To have a hardware platform with similar features to those existing in an industrial environment. This way, students can practice concepts such as: adjustment of PID controllers, programming of PLCs (Programming Logic Controllers), industrial communications and adjustment of inverter's parameters.
- To provide students a remote access to this hardware platform in order to perform experiments from home.

- The remote laboratory must be an environment for selflearning. For this objective, the system detects possible errors and offers the necessary feedback to the user. Also, it provides information about which aspects should be considered to solve these errors.

- To have a self-assessment system. The system automatically establishes different operating modes to evaluate all the cases that may occur in a real industrial system.

This application proposed has been integrated a blendedlearning educational methodology. This laboratory, with the features above commented, provides an improvement in the learning process of the student to be this active, self-directed, and constructive (Shuell, 1986). This issue is demonstrated throughout this paper.

This paper is structured as follows: the next section describes the system, including the industrial system emulated, the remote laboratory and the client interface of the application. Section 3 explains an experimental example and some educational results obtained. Finally some conclusions are reported in Section 4.

\section{OVERALL SYSTEM DESCRIPTION}

This section is an introductory description of the remote platform developed. The industrial system emulated by the application is described in subsection 2.1. Next, subsection 2.2 shows the hardware model developed, which emulates the real pumping system. Subsequently, subsection 2.3 shows the hardware and software architecture of remote laboratory. In 
subsection 2.4, the client application is described, focusing the explanation in how students can load the program into the PLC, display the status of the plant and be able to adjust certain parameters. Finally, subsection 2.5 shows the selfassessment system.

\subsection{Industrial system emulated}

In order to practice the concepts previously commented (adjustment of PID controllers, programming of PLCs, industrial communications and adjustment of inverters), a remote laboratory of a hardware model was developed. The hardware application emulates the real case of a pumping station control. In particular, the real system selected is a water distribution system in an industrial plant. This pumping system has a total of 4 pumps model ESPA HX210 150 (www.espa-argentina.com) and an inverter type OMRON MX2 (www.omron.com). The real pumping system must be able to adapt at any demand using the 4 pumps, with the only inverter. For this objective, a pressure sensor is considered in range of 0-10 bars, traduced to 4-20mA. The final objective of the system is to keep stable a pressure of 6 bars in the industrial plant. To do this, one of the pumps must be controlled by the inverter with the output frequency needed to achieve pressure of 6 bars. If the case of the demand could not be achievable with only one pump controlled by the inverter, one or more of the remaining pumps could be directly connected to the three-phase power line connection by means of contactors managed by a PLC. In the case of an increase of the pressure (decrease in demand) pumps must be disconnected from the three-phase line. If there is not demand, the pump controlled by the inverter will be also disconnected. In addition, in case of damaging any pump of the installation, the system must continue working with the same benefits but at $75 \%$ of its performance. Furthermore, the system must control that all the pumps became equally older.

With this industrial system emulated, students can practice different control concepts:

- To set the PID parameters. A PID controller of the inverter is in charge of controlling the output frequency needed to maintain the pressure desired.

- PLC programming. A PLC will be in charge of turning on and turning off the needed pumps to ensure the demand, so it is necessary to implement the control program of the PLC depending on the pressure.

- Industrial communications. The PLC must communicate with frequency inverter to control several things, such as turning on and off the device.

- To set inverter parameters. It is necessary to set ramps of start, stop, times, modes of operation, etc. (in addition to the previously commented PID controller parameters).

Obviously, students do not access to a real system of these features. As related, it has been designed a hardware model with similar functionality. This model will allow practicing all the above commented concepts and the described in the next section.

\subsection{Hardware model of the real system}

The hardware model is made up of two parts. The first part is shown in Figure 1 and it contains the following components:

\section{A PLC type CP1L-J from OMRON.}

\section{An Inverter MX2.}

3. A three-phase motor of 4 magnetic poles.

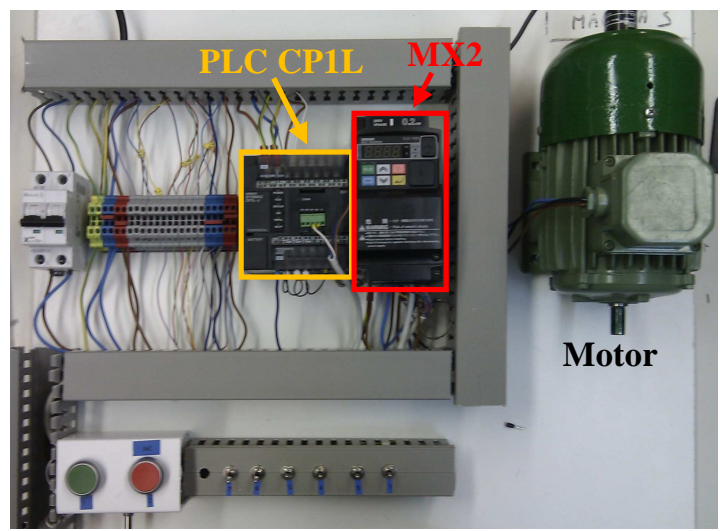

Fig. 1. First part of the hardware model.

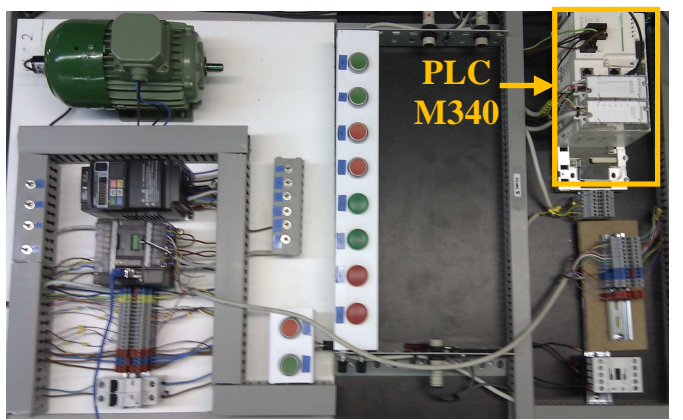

Fig. 2. Complete hardware model.

As indicated in Section 2.1, the hardware model can use up to 4 pumps with the objective of maintaining a stable pressure of 6 bars regardless of the demand. The outputs $0-3$ of the CP1L-J are used to indicate the pump controlled by the inverter (only one pump can be controlled at the same time) and the outputs 4-7 to indicate the active pumps directly connected to the three-phase line (at $100 \%$ of their power).

This part of the hardware model is connected to a PLC type Modicon M340 from Schneider (www.schneiderelectric.com), which is in charge of simulating the industrial plant (Figure 2). This device runs a program that is in charge of interpreting the signals coming from the CP1L-J, such as the pump controlled by the inverter and the active pumps. An analog input of the PLC M340 is employed to read the speed of the engine controlled by the inverter (in percentage value). The program of this equipment produces as a result the 
simulated value from the pressure sensor with is calculated from the pumps (controlled and active pumps). This value of the transducer is sent through an analog signal directly to the MX2 inverter, so it is possible to use it as feedback input for the inverter PID controller. In contrast with the CP1L-J, the program of the PLC M340 is not designed by the student.

In addition, a potentiometer is connected to the PLC M340 in order to enable the manual selection of a demand. It is also possible to load different profiles to PLC M340 where the demand changes in real time. These experiments aim to evaluate the following concepts of operation in students:

- The proper power on / off pumps.

- The adjustment of PID controller of the inverter.

- The adjustment of inverter parameters such as ramps of acceleration, minimum and maximum frequencies, etc.

\subsection{The remote laboratory}

The implementation of the remote laboratory is made up for a completed development of different software and hardware elements to provide remote functionalities to students in order to carry out the activities described above. In this way, students can load their programs to the PLC CP1L-J which is in charge of controlling the system. Moreover, it is possible to modify the configuration of the MX2 inverter. For that purpose, this device is connected to a web server via USB connector, so that this server is able to load programs on the device. To do this, the CP1L-J has a generic program loaded which is modified by the web server depending on the configurations established by the program developed by students. In this way, the system guarantees that a correct program is executed by the CP1L-J and possible errors are indicated to the student.

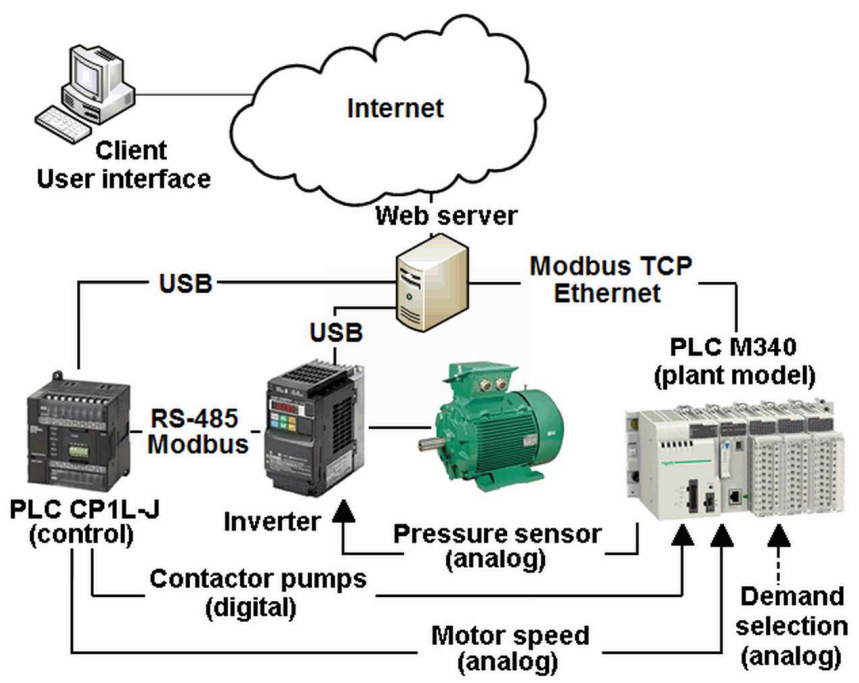

Fig. 3. Complete hardware model.

The PLC M340 is connected through Ethernet to the aforementioned web server (Figure 3). Through this connection, the server is able to send commands that modify certain memory parameters in the PLC, which are interpreted as orders according to the program included in the device.
The main purpose of this functionality is to generate a few demand/time profiles in the plant, which allows a further automatic correction of the exercises carried out by the students. At the same time, through this connection, the server monitors the status of the simulated plant: state of the pumps connected to the inverter, state of the pumps connected to the three phase line, pumps in error state, demand, pressure transducer and motor speed (which simulate the controlled pump speed). With this information, it is generated a chart in the user interface, which is shown to students, so they can follow the evolution of the plant under the control program loaded. In addition, the server reflects the evolution in time of the different parameters of the given profile, and generates several graphs that will be sent to the students. Then, students can analyse the response of their programs and fix the errors found in the response.

For a correct access of students to the remote plant, there are two critical services within the server: access restricted to registered students (with user name and password) and a booking system, so that only one user can try to load the program and try to do the exercises. This booking system is a program that assigns a schedule (day and time) so the student can enter the system remotely through the Internet and perform the practical exercises.

As already mentioned, the remote laboratory has a user interface to view the status of the PLC variables, whose function is to have a graphical representation of the industrial plant state. This interface is shown in Figure 4. With this application, students can view the following information:

- Demand of the plant. As indicated above, this demand can be established with a demand profile.

- The value of the transducer or sensor.

- Frequency of the speed controller or inverter. It is the number of litres supplied by the pump being controlled by the inverter. This value gives an idea of the percentage of pump used (from 0 to 1088).

- Indicators P1, P2, P3 and P4 represent the pumps. When the symbol is green, it indicates that the pump is running. The symbol is represented in red if an error has occurred.

- The black lines indicate connections to the MX2 inverter pumps (only one pump can be controlled by the inverter).

- The red lines indicate connections to the three-phase line connection. In this case, there may be zero or more pumps connected to the three-phase line (other than which is connected to the speed inverter).

\subsection{The client interface}

The client application was developed using the software Easy Java Simulations (EJS) (Esquembre, 2004). EJS is a program developed in Java, specifically designed for the creation of interactive dynamic simulations. EJS is oriented to people (teachers and/or students) who do not know or are not experts in programming. Users can create in a simple way dynamic 
simulations, since this software is in charge of generating almost all the code needed for animation. EJS is completely implemented in Java, so it provides total portability of generated applications. EJS automatically generates the simulation compiled Java code, packaging the resulting files to a compressed file, and generates HTML pages containing embedded virtual applet-shaped lab.

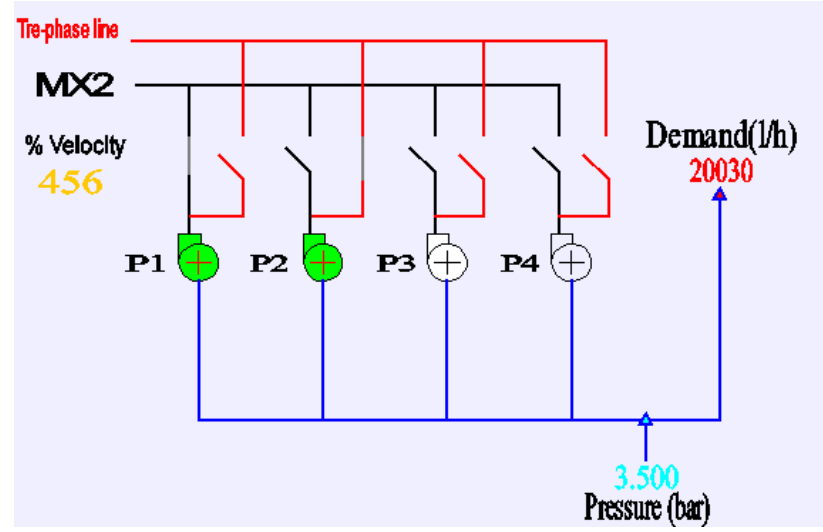

Fig. 4. Virtual interface which shows the status of the plant.

Figure 5 shows two images of the developed client interface. On the one hand, there is a virtual panel, which shows the status of pumps and the main parameters of the remote plant. This virtual part is the interface shown in Figure 4, which monitors the status of the plant. On the other hand, there is a remote interface via a web cam of the real state of the hardware model. In addition, inside the panel has been included more information using of the plant through an augmented reality interface. Under this application, students can load remotely the control program into PLC CPL1-J. In addition, the right side of the interface displays interactive exercises that students have to perform remotely. Such practical and interactive exercises are explained in more detail in the next section.

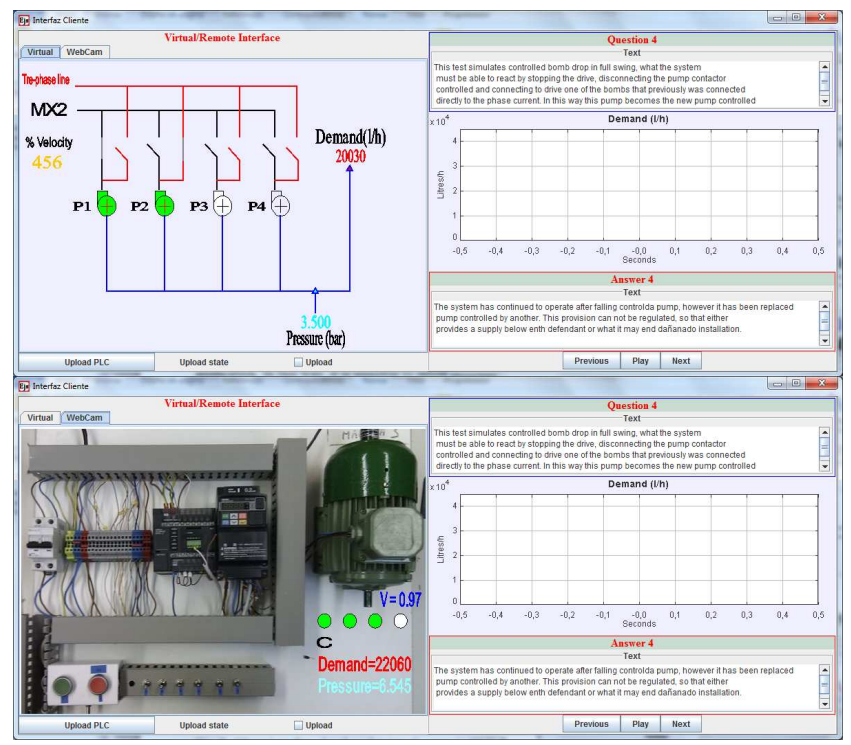

Fig. 5. Client interface developed (virtual - remote GUI).

\subsection{Interactive exercises and self-assessment system}

For self-evaluation of students' knowledge, a series of interactive exercises have been added in the client application. In this way, it is possible to determine the level of acquired knowledge and the students can evaluate their self since they receive guidelines, automatically, depending on the type of answer given to each exercise. Thus, this series of interactive exercises is able to provide the correct answer, indicate where the student has been wrong or indicate according to responses which part of the theoretical concepts has to revise.

This series of interactive educational guides have as main objective implementation in practice of theoretical concepts on a real system. By the client and remote application, the students have the possibility of putting them into practice on a real system using the hardware model.

For students' assessment, there are prepared various tests. These tests introduce a demand curve, which evolves on time and monitor the reactions of the system. To determine if overall performance is adequate, the system keeps track of the pressure value on the transducer, and checks that it does not exceed a maximum level of error that this can offer with respect to the desired 6 bars. In addition, other requirements have to be reached such as the power of the pumps process and details such as sending communications of RUN/STOP commands.

In Figure 6, it can be seen different profiles from the four proposed tests. The first test is designed to check a general operation of the system. This introduces an ascending ramp followed by a descending ramp and checks the output pressure of about 6 bars which must keep stable in the system. In addition, in this exercise, students must program the appropriate order of the commands RUN and STOP. The second test checks the operation of the system after the fall of one of the pumps which is directly contacted to the three phase line.
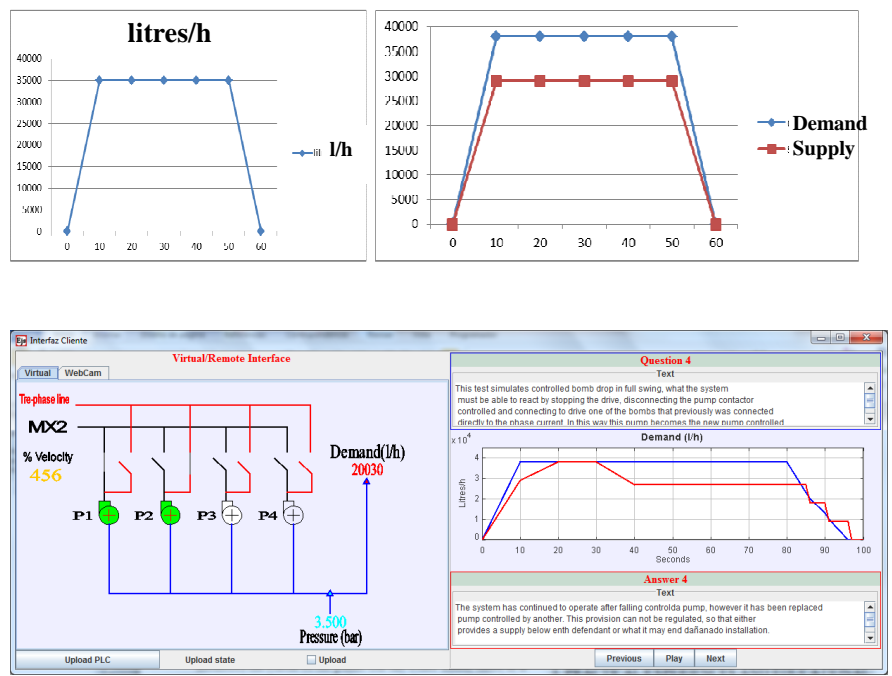

Fig. 6. Different tests for evaluating student's work 


\section{PRACTICAL EXPERIENCES AND EDUCATIONAL RESULTS}

\subsection{Practical example}

In this section, a trace of the system for the first of the tests described in Section 2.5 is presented. In this trace, the initial demand of the system is zero. This demand will progressively increase until $35 \mathrm{~m}^{3} / \mathrm{h}$. Once this demand is achieved, this value is maintained during 40 seconds and subsequently decreases until zero. The proposed system uses a PID controller in the MX2 inverter in order to assure a pressure of 6 bars during all the experiment. At the beginning of the experiment, the system connects the first pump when the demand begins (pump 1 in the example). This first pump will be the one controlled by the PID of the inverter in order to allow the system regulate the pressure. In this section, the values of the digital outputs of the PLC are illustrated by using the table 1:

\begin{tabular}{|l|c|c|c|c|c|c|c|c|}
\hline & \multicolumn{4}{|c|}{ Controlled pump } & \multicolumn{4}{c|}{$\begin{array}{c}\text { Pumps to the three } \\
\text { phase network }\end{array}$} \\
\hline Output & 0 & 1 & 2 & 3 & 4 & 5 & 6 & 8 \\
\hline Value & 1 & 0 & 0 & 0 & 0 & 0 & 0 & 0 \\
\hline
\end{tabular}

Table 1. Table employed to illustrate the values of the PLC digital outputs.

As it is previously indicated, the inverter implements a PID controller in order to maintain a constant pressure. The feedback of the PID is the measurement obtained from a pressure transductor connected to the OI terminal of the inverter (4 to $20 \mathrm{~mA}$ ). $4 \mathrm{~mA}$ corresponds with 0 bars and 20 $\mathrm{mA}$ with 10 bars (maximum pressure). The PID reference (6 bars) is indicated in $\mathrm{Hz}$ using a register of the inverter (the working rank of the inverter is from 0 to $50 \mathrm{~Hz}$ ). Using the configuration established by the student, the program in the CP1L-J PLC employs Modbus to read the pump frequency (controlled pump). When the frequency is $50 \mathrm{~Hz}$ during several seconds the demand is greater than the maximum that the pump can provide. Therefore, it is necessary to activate a new pump. In this example, the pump 2 is connected to the three-phase network (see Table 2).

\begin{tabular}{|l|c|c|c|c|c|c|c|c|}
\hline & \multicolumn{4}{|c|}{ Controlled pump } & \multicolumn{4}{c|}{$\begin{array}{c}\text { Pumps to the three } \\
\text { phase network }\end{array}$} \\
\hline Output & 0 & 1 & 2 & 3 & 4 & 5 & 6 & 8 \\
\hline Value & 1 & 0 & 0 & 0 & 0 & 1 & 0 & 0 \\
\hline
\end{tabular}

Table 2. Example. Iteration 1.

Considering the profile demand previous indicated for this example, after some time, the same thing will occur, i.e., the controlled pump has an output frequency of $50 \mathrm{~Hz}$. Therefore, a new pump (pump 3) must be connected to the three-phase network. The controlled pump continues being the pump 0 (see Table 3 ).

\begin{tabular}{|l|c|c|c|c|c|c|c|c|}
\hline & \multicolumn{4}{|c|}{ Controlled pump } & \multicolumn{4}{c|}{$\begin{array}{c}\text { Pumps to the three } \\
\text { phase network }\end{array}$} \\
\hline Output & 0 & 1 & 2 & 3 & 4 & 5 & 6 & 8 \\
\hline Value & 1 & 0 & 0 & 0 & 0 & 1 & 1 & 0 \\
\hline
\end{tabular}

Table 3. Example. Iteration 2.

After some time, the same situation will occur; therefore, a new pump is connected to the three-phase network (pump 4, see Table 4).

\begin{tabular}{|l|c|c|c|c|c|c|c|c|}
\hline & \multicolumn{4}{|c|}{ Controlled pump } & \multicolumn{4}{c|}{$\begin{array}{c}\text { Pumps to the three } \\
\text { phase network }\end{array}$} \\
\hline Output & 0 & 1 & 2 & 3 & 4 & 5 & 6 & 8 \\
\hline Value & 1 & 0 & 0 & 0 & 0 & 1 & 1 & 1 \\
\hline
\end{tabular}

Table 4. Example. Iteration 3.

As previously indicated, after 40 seconds, the demand begins to decrease (therefore an increase of the pressure is obtained). When the frequency is $0 \mathrm{~Hz}$ during several seconds, the program implemented by the student must detect that an over-pressure is generated. Therefore, the program must to progressively deactivate the pumps connected to the threephase network. The pumps must be deactivated in the same order that they were activated in order to equalize the use of the pumps. Considering this example, after 40 seconds the new state will be the one illustrate in Table 5 .

\begin{tabular}{|l|c|c|c|c|c|c|c|c|}
\hline & \multicolumn{4}{|c|}{ Controlled pump } & \multicolumn{4}{c|}{$\begin{array}{c}\text { Pumps to the three } \\
\text { phase network }\end{array}$} \\
\hline Output & 0 & 1 & 2 & 3 & 4 & 5 & 6 & 8 \\
\hline Value & 1 & 0 & 0 & 0 & 0 & 0 & 1 & 1 \\
\hline
\end{tabular}

Table 5. Example. Iteration 4.

This situation will continue until all the pumps connected to the three-phase network are deactivated (first the pump 3 and second the bomb 4). Finally, when the demand decreases until the controlled pump must be also deactivated (the pump 1 in the example), by stopping the inverter first and then deactivating the pump. Therefore, the student must to develop a program in the PLC CP1J-L which must take into account the following aspects that are considered in the selfassessment system:

- Initially, the first pump is not activated until the measured pressure is lower than the reference.

- The PLC must not activate the inverter before to connect the first pump.

\subsection{Analysis of the self-assessment system}

This remote application is being used in the subject "Control Systems" taught in the Automatics and Robotics Master's degree at the University of Alicante (www.mayr.ua.es). 
To evaluate the results obtained with the self-assessment system, an evaluation was obtained from the students. They were divided into 2 groups, from a total of 20 students. The first group (group A) performs scheduling and system tuning in a traditional way. That is, this group of 10 students moving to college and they take turns doing the programming model and the subsequent adjustment of the various parameters that configure the correct behaviour. Using the interface described in Section 2.3, they could verify themselves the correct system operation. Although they have the support of the teacher, the students loaded the program in the model and should monitor compliance all possible operating conditions specified in practice.

The second group (group B) also consists of 10 students but, in this case, it makes use of the proposed remote virtual laboratory. Thus, they did not need to go to the University for testing the system operation. Some of them are people who have problems to attend classes due to working issues. This application gives them flexibility to schedule in testing developments and adjustments. This latter group also makes use of the proposed self-evaluation system. Thus, once students developed programs and did an initial adjustment of the model, they have to use the interactive tutorials and statements described in Section 2.4 to verify the correct behaviour and completely set the parameters and possible operating conditions. To make a comparison between both groups, the following criteria were selected:

- PID settings. It checks if the PID controller introduced in the inverter allows proper scope of the desired pressure. This evaluates if the three parameters for the controller are correct and allow adequate time response.

- Code optimization. This criterion is used to determine if the code implemented in the controller is optimized.

- Terms of start / stop. It is checked if different pumps are turned on or off in the correct sequence.

- Speed of response. This issue determines if the response speed is suitable for different profiles.

- Faults detected by the automatic evaluation system. The evaluation system is responsible for entering, automatically, different demand profiles. Under these operating conditions, the system checks various aspects, such as if the drive is connected before starting it.

As shown in Figure 8, it is observed that the marks of both groups are quite high in general. This confirms the correct understanding of the problem. Analysing more in detail the scores of both groups on each of the criteria discussed above, additional conclusions can be drawn. Firstly, in almost all aspects evaluated was obtained a high score in the case of Group B than in the case of Group A. Where there are differences is most notable in terms of criteria start / stop and fault automatic evaluation system. Both are described in detail in the teaching guides employed by Group B. In addition, the self-assessment system places special emphasis on checking these criteria together with the proper adjustment of the PID controller. This has led to obtain an improvement in the ratings of Group B. Only code optimization criterion is similar in both cases. Analysing the reason for this last fact leads to the conclusion that the latter is not treated in detail in the teaching guides employed by Group B.

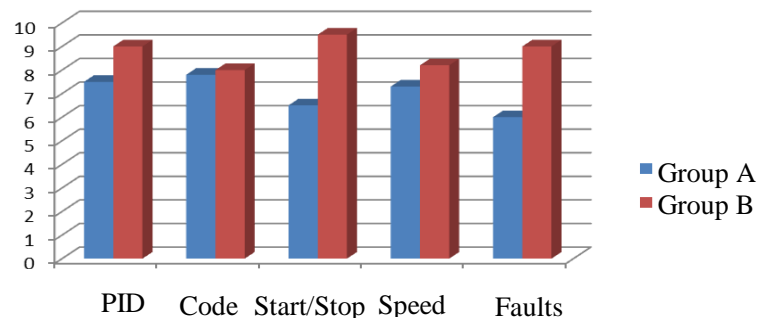

Fig 8. Comparison between students' marks who did not use the remote virtual laboratory (A) and those that used it (B).

\section{CONCLUSIONS AND FUTURE WORK}

A remote laboratory employed in the practical learning of the subject "Control Systems" taught in the Automatics and Robotics Master's degree at the University of Alicante, has been described. The application presented allows students to practice remotely different theoretical concepts using of a hardware model of a real industrial process about a pumping system. The use of the proposed tool allows reinforcing students' active learning since they can perform practical exercises with the aim of understanding the knowledge acquired. In addition, students can understand the scope of theoretical concepts by applying them to a realistic industrial environment. Educational results have been positive and students have achieved an improvement in learning.

\section{ACKNOWLEDGMENT}

This work is supported by the program "Technologic \& Educative Research Groups" of the University of Alicante.

\section{REFERENCES}

Clausen, T. (2005). Undergraduate engineering education challenged by the Bologna declaration. IEEE Transactions on Education, 48 (2), 213-215.

Esquembre, F. (2004). Easy Java Simulations: A software tool to create scientific simulations in Java. Computer Physics Communications, 156(2), 199-204.

Jara, C. A.; Candelas, F.A; Puente, S. T., Pomares, J., Torres, F. (2009). Practical experiences using RobUALab.ejs: a virtual and remote laboratory for Robotics e-learning. 9th IFAC Symposium on Advances in Control Education. Kumamoto (Japan).

Jara, C. A.; Candelas, F. A.; Gil, P.; Torres, F.; Esquembre, F.; Dormido, S. (2011) Ejs+EjsRL: An interactive tool for industrial robots simulation, Computer Vision and remote operation. Robotics and Autonomous Systems, 59 (6). 389-401.

Shuell, T.J. (1986). Cognitive Conceptions of Learning. Review of Educational Research, 56(4), 411-436. 